\title{
EVALUACIÓN DE LA CAPACIDAD AMORTIGUADORA DE INGREDIENTES UTILIZADOS EN LA FORMULACIÓN DE DIETAS PARA RUMIANTES ${ }^{1}$
}

\author{
Oziel D. Montañez Valdez ${ }^{2}$, Ricardo Barcena Gama ${ }^{3}$, Sergio S. González Muñoz ${ }^{3}$, María Esther Ortega Cerrilla ${ }^{3}$, \\ Mario A. Cobos Peralta ${ }^{3}$, Juan H. Avellaneda Cevallos 4
}

\begin{abstract}
RESUMEN
Evaluación de la capacidad amortiguadora de ingredientes utilizados en la formulación de dietas para rumiantes. Se recolectaron 22 muestras de diferentes ingredientes, que se clasificaron en: energéticos altos $(>15 \%)$ y bajos $(<$ $15 \%$ ) en proteína, forrajes, alimentos fermentados y aditivos, con el objetivo de evaluar su capacidad amortiguadora (CA). Se determinó la capacidad amortiguadora expresando los resultados en miliequivalentes de ácido clorhídrico requerido para producir un cambio en una unidad de $\mathrm{pH}$ de la muestra original de alimento. Los resultados obtenidos fueron comparados con los reportados en otros estudios. La CA es alta en alimentos altos en proteína y leguminosas, intermedia en alimentos energéticos y fermentados, y baja en gramíneas.
\end{abstract}

Palabras clave: Capacidad amortiguadora, alimentos, acidosis, rumiantes.

\begin{abstract}
Evaluation of the buffering capacity of ruminant feed-stuffs used for diets formulation. Twenty two different energy ingredients, high $(>15 \%)$ and low $(<15 \%)$ in protein, forage, fermented feeds and additives, were evaluated for their buffering capacity (BC). The buffering capacity was determinated by expressing the data on milliequivalents of chloridric acid required to produce a unit change in $\mathrm{pH}$ from the original feed sample. The results obtained were compared with those from other studies. The $\mathrm{BC}$ was highest for high protein feeds and legume forages, intermediate for energy and fermented feeds, and lowest for grass forages.
\end{abstract}

Key words: Buffering capacity, feedstuffs, acidosis, ruminant.

\section{INTRODUCCIÓN}

Las dietas altas en concentrado contienen grandes cantidades de carbohidratos rápidamente fermentables, lo cual trae como consecuencia una serie de problemas o trastornos metabólicos: baja en $\mathrm{pH}$ ruminal, acidosis, laminitis, menor producción de grasa y leche, reducción en la digestibilidad de la fibra, problemas que impactan directamente en la eficiencia productiva de los animales y, por consecuencia, el margen de ganancia de una explotación pecuaria (Nocek 1997; Owens et al. 1998; Garrett et al. 1999; Oetzel 2001).

Para contrarrestar estos efectos adversos, se ha generado una serie de productos como los ionóforos, antibióticos y sustancias amortiguadoras. En el caso de las

1 Recibido: 30 de setiembre, 2005. Aceptado: 1 de febrero, 2006. Trabajo elaborado como parte de tesis doctoral.

2 División de Bienestar y Desarrollo Regional. Departamento de Desarrollo Regional. CUSUR. Universidad de Guadalajara. Av. Prolongación Colón S/N. Carretera Libre Ciudad Guzmán-Guadalajara Km. 1. C.P. 49000. CD. Guzmán, Municipio de Zapotlán El Grande. Jalisco, México. Tel +52 3415752222 Ext. 6085. Correo electrónico: montanez77@hotmail.com; oziel.montanez@cusur.udg.mx

3 Colegio de Postgraduados. Programa de Ganadería. Carretera México-Texcoco Km. 36.5. C. P. 56230 Montecillo, Texcoco, Estado de México, México. Tel. (595) 9520200 Ext. 1727.

4 Unidad de Investigación. Facultad de Ciencias Pecuarias. Universidad Técnica Estatal de Quevedo. Km. 1 vía Santo Domingo. Quevedo, Los Ríos, Ecuador. 
sustancias amortiguadores se ha encontrado que pueden afectar las condiciones ruminales, mejorando su $\mathrm{pH}$, lo que aumenta los niveles de producción animal. Sin embargo, la respuesta encontrada cuando son adicionados a dietas altas en concentrado ha sido variable, ya que en algunos estudios hubo mejor respuesta animal, pero en otros no, lo cual se debe posiblemente a factores como la cantidad y tipo de amortiguador empleado, o por no tomar en cuenta la capacidad amortiguadora intrínseca (CA) de los ingredientes utilizados en la dieta (Staples y Lough 1989; Sear y Walsh 1998).

Jasaitis et al. (1987) define la capacidad amortiguadora como la cantidad de ácido o base requerida para producir una cambio en una unidad de $\mathrm{pH}$ de una muestra de alimento. Cada ingrediente presenta una CA distinta que puede afectar la acción del amortiguador, y que al no tomarse en cuenta la respuesta animal puede no ser la esperada. Por lo anterior, el objetivo del presente estudio fue evaluar la capacidad amortiguadora de algunos ingredientes utilizados en la alimentación de bovinos.

\section{MATERIALES Y MÉTODOS}

Se recolectaron 22 muestras de alimentos utilizados comúnmente en la formulación de dietas para ganado bovino. Para cada ingrediente se determinó la CA de acuerdo a lo descrito por Jasaitis et al. (1987), el cual consiste en tomar $5 \mathrm{~g}$ de la muestra de alimento y suspenderla en $50 \mathrm{ml}$ de agua destilada desionizada a $\mathrm{pH} 7$ y mezclar continuamente con una barra magnética. Después de tres minutos de equilibrio se registró el pH inicial con un potenciómetro portátil (ORION modelo SA 210). Posteriormente se tituló con ácido clorhídrico $(0,1 \mathrm{~N})$ hasta que el $\mathrm{pH}$ disminuyó a 4 . El volumen total de ácido agregado a cada muestra fue registrado y posteriormente multiplicado por la normalidad del $\mathrm{HCl}$, siendo estos los miliequivalentes de ácido necesarios para bajar el pH inicial de la muestra a 4. La CA se calculó por la división de los miliequivalentes sobre el cambio total de unidades de $\mathrm{pH}$ (del $\mathrm{pH}$ inicial a 4). La CA se expresó en miliequivalentes de ácido requerido para producir un cambio en una unidad de $\mathrm{pH}$ de una muestra de alimento. Se realizaron tres repeticiones por cada alimento, los forrajes utilizados fueron picados a 2 $\mathrm{cm}$ y los alimentos restantes en su presentación comercial. Los análisis fueron realizados en el laboratorio de nutrición animal ubicado en la granja experimental del Colegio de Postgraduados, localizado en la carretera México-Texcoco, km 36,5, en Montecillo, Municipio de Texcoco, Estado de México.

\section{RESULTADOS Y DISCUSIÓN}

Los resultados obtenidos se compararon con los publicados por Jasaitis et al. (1987) y Peña et al. (2001) (Cuadro 1).

Al comparar la CA natural del presente estudio con los observados por Peña (2001) se observan similitudes entre ellos como por ejemplo el sorgo, trigo, salvado de trigo o melaza, sin embargo, se observó que en algunos ingredientes la diferencia en CA fue considerable entre lo encontrado por Jasaitis et al. (1987) y Peña (2001) (eg. avena, salvado de trigo y las harinas de pescado y carne). Se ha observado que la CA natural es más baja $\left(<70\right.$ meq x $\left.10^{-3}\right)$ para los alimentos energéticos, intermedia para gramíneas y alimentos con 15 a $35 \%$ de proteína cruda (>71 meq x $10^{-3}$ y $<100$ meq x $10^{-3}$ ), y alta (> 100 meq $\times 10^{-3}$ ) para leguminosas y alimentos con más de $35 \%$ de proteína cruda, posiblemente debido a la presencia de grupos amino (Van Soest et al. 1984; Jasaitis et al. 1987; Canale y Stokes 1988). En el caso de los alimentos fermentados, ya sea solos o combinados, su capacidad amortiguadora dependerá del tipo de forraje utilizado y de la adición de minerales o aditivos (Crawford et al. 1983).

Estas diferencias se pueden atribuir a factores propios del alimento empleado, como la capacidad de intercambio de iones, y que la fibra presenta una CA variable pero substancial; por ejemplo, las leguminosas (específicamente la alfalfa) incrementan el $\mathrm{pH}$ ruminal y CA del rumen; la capacidad de intercambio de cationes es alta en la fibra de la alfalfa y bajo en el ensilado de maíz (Mertens, 1979; Van Soest y Jones, 1980; Van Soest et al 1984; Jasaitis et al. 1987; Sniffen y Robinson 1987; Le Ruyet et al. 1992). Otro factor importante es el contenido de minerales en el alimento, el cual varía según su origen, animal o vegetal, el procesamiento, el lugar de donde proviene, factores climáticos, edafológicos, etc. (Jasaitis et al. 1987).

Factores propios del mismo análisis influyen también sobre la variación de la CA, por ejemplo, las diferencias en el tamaño de la muestra tratada, el tipo y volumen del líquido usado para suspender la muestra, el tipo de ácido o base usado en la titulación, el punto final de $\mathrm{pH}$, el tipo de alimento titulado y la manera en la cual se expresaron los resultados (Wohlt et al. 1987). Tomando en cuenta los factores anteriores y algunos otros como el tamaño de partícula del alimento y su procesamiento, la capacidad amortiguadora de una dieta puede variar considerablemente, obteniéndose así diferencias en la respuesta animal, dada la diferencia en la capacidad amortiguadora natural de los ingredientes usados en la dieta. 
Cuadro 1. Capacidad amortiguadora natural de algunos ingredientes utilizados en dietas para rumiantes. Colegio de Postgraduados, Montecillo, Estado de México, México. 2004.

\begin{tabular}{|c|c|c|c|c|c|c|}
\hline \multirow{2}{*}{ Ingredientes } & \multicolumn{2}{|c|}{ Presente estudio } & \multicolumn{2}{|c|}{ Jasaitis et al. 1987} & \multicolumn{2}{|c|}{ Peña et al. 2001} \\
\hline & $\mathbf{C A}^{1}$ & $\mathbf{p H i}^{2}$ & CA & pHi & CA & pHi \\
\hline \multicolumn{7}{|l|}{ Energéticos } \\
\hline Avena (Avena sativa) & 33,00 & 6,83 & 63,00 & 5,25 & 21,97 & $\ldots$ \\
\hline Cebada (Hordeum vulgare) & 25,23 & 6,47 & 34,00 & 5,73 & $\ldots$ & $\ldots$ \\
\hline Maíz (Zea mays) & 16,59 & 6,51 & 39,00 & 5,05 & 31,22 & $\ldots$ \\
\hline Sorgo (Sorghum vulgare) & 16,60 & 7,14 & 27,00 & 6,40 & 17,39 & $\ldots$ \\
\hline Trigo (Tritucum vulgare) & 23,30 & 7,12 & 19,00 & 6,32 & 19,43 & $\ldots$ \\
\hline Salvado de trigo & 46,57 & 6,90 & 89,00 & 6,23 & 40,58 & $\ldots$ \\
\hline Melaza & 84,12 & 5,98 & $\ldots$ & $\ldots$. & 86,58 & $\ldots$ \\
\hline \multicolumn{7}{|l|}{ Forrajes } \\
\hline Alfalfa (Medicago sativa) & 134,00 & 6,49 & 124,00 & 6,28 & 170,60 & $\ldots$ \\
\hline Ovillo (Dactylis glomerata) & $\ldots$ & $\ldots$ & 99,00 & 5,77 & $\ldots$ & $\ldots$ \\
\hline Paja de avena & 49,10 & 8,24 & $\ldots$ & $\ldots$ & 55,26 & $\ldots$ \\
\hline Rastrojo de maíz & 54,34 & 7,64 & $\ldots$ & $\ldots$ & 35,20 & $\ldots$ \\
\hline \multicolumn{7}{|l|}{ Altos en proteína } \\
\hline Harina de carne & 181,24 & 6,24 & 231,00 & 6,76 & 98,63 & $\ldots$ \\
\hline Harina de pescado & 116,47 & 5,45 & 390,00 & 5,97 & 48,01 & $\ldots$ \\
\hline Harina de sangre & 106,31 & 6,35 & $\ldots$ & $\ldots$ & 23,76 & $\ldots$ \\
\hline Pasta de soya (Glicyne max) & 110,30 & 7,02 & 130,00 & 6,65 & 84,91 & $\ldots$ \\
\hline Pasta de coco (Cocos nucifera & a) $\quad \ldots$ & $\ldots$ & $\ldots$ & $\ldots$ & 80,21 & $\cdots$ \\
\hline Urea & $\ldots$ & $\ldots$ & 3,00 & 5,85 & 12,25 & $\ldots$ \\
\hline \multicolumn{7}{|l|}{ Fermentados } \\
\hline Gluten de maíz & 16,50 & 6,47 & 0,00 & 4,05 & $\ldots$ & $\ldots$ \\
\hline Ensilado de maíz & 86,70 & 5,82 & 0,00 & 3,94 & 97,40 & $\ldots$ \\
\hline \multicolumn{7}{|l|}{ Aditivos } \\
\hline Premezcla mineral ${ }^{3}$ & 534,87 & 9,43 & $\ldots$ & $\ldots$ & 315,37 & $\ldots$ \\
\hline Bicarbonato de sodio & 2005,52 & 8,90 & 1521,00 & 8,11 & 1960,52 & $\ldots$ \\
\hline \multicolumn{7}{|c|}{1 Capacidad amortiguadora en meq x $10^{-3}$} \\
\hline \multicolumn{7}{|c|}{$2 \mathrm{pH}$ inicial de la muestra original } \\
\hline \multicolumn{7}{|c|}{$\begin{array}{l}3 \text { Rumisal Plus por cada kg contiene: Ca } 130 \text { g; P } 50 \text { g; Na } 109 \text { g; Cl } 200 \text { g; Fe 4,30 g; } \\
\text { Mg 3,33 g; Mn } 200 \text { mg; Cu } 80 \text { mg; Co } 66,6 \text { mg; I } 4 \text { mg; Zn } 80 \text { mg; Se } 70 \text { mg. }\end{array}$} \\
\hline
\end{tabular}

Es importante destacar que todos los ingredientes poseen una CA potencial, la cual debe ser tomada en cuenta al adicionar amortiguadores a las dietas. Si se considera la CA de los ingredientes de la dieta, considerando que ésta es aditiva, la adición de amortiguadores sería más efectiva. Por tanto, al tomar esto en consideración haría posible determinar qué tipos de dietas requieren ser amortiguadas y cuál sería la concentración más adecuada del amortiguador a utilizar.

\section{CONCLUSIONES}

De acuerdo con las condiciones del presente estudio se concluye:
La capacidad amortiguadora natural de los ingredientes fue alta (> 100 meq x 10-3) en alimentos altos en proteína y en leguminosas (harina de carne y alfalfa) $(>15 \%)$, media $\left(>52\right.$ meq x $10^{-3}<98$ meq x $\left.10^{-3}\right)$ en alimentos fermentados y energéticos (ensilado de maíz y melaza) y baja $\left(<52\right.$ meq x $\left.10^{-3}\right)$ en gramíneas (maíz, sorgo, cebada).

\section{LITERATURA CITADA}

CANALE, C. J.; STOKES, M. R. 1988. Sodium bicarbonate for early lactation cows fed corn silage or hay crop silage-based diets. Journal of Dairy Science 71:373-380. 
CRAWFORD, R. J.; SHRIVER, B. J. G.; VARGA, A.; HOOVER, W. H. 1983. Buffer requirements for maintenance of $\mathrm{pH}$ during fermentation of individual feeds in continuous cultures. Journal of Dairy Science 66: 1881-1890.

GARCÍA, E. 1987. Modificaciones al sistema de clasificación climática de Koeppen para adaptarlo a las condiciones de la República Mexicana. 4 ed. UNAM, México. p. 65-79.

GARRETT, F. E.; PEREIRA, N. M.; NORDLUND, V. K.; ARMENTANO, E. L.; GOODGER, J. W.; G. R. OETZEL. 1999. Diagnostic methods for the detection of subcute ruminal acidosis in dairy cows. J. Dairy Sci. 82:1170-1178.

JASAITIS, D. K.; WOHLT, J. E.; EVANS, J. L. 1987. Influence of feed ion content on buffering capacity of ruminant feedstuffs in vitro. Journal of Dairy Science 70: 1391-1403.

LE RUYET, P.; TUCKER, W. B.; J. F. HOGUE.; ASLAM, M.; LEMA, M.; SHIN, S.; MILLER T. P.; ADAMS, G. D. 1992. Influence of dietary fiber and buffer value index on the ruminal milieu of lactating dairy cows. Journal of Dairy Science 75: 2394-2408.

MERTENS, D. R. 1979. Effect of buffers upon fiber digestion. In: Hale, W. H.; Meinhardt, P. eds. Regulation of Acid-Base Balance. Piscataway, N.J. Church \& Dwight Company, Inc. USA. p. 65-76.

NOCEK, J.E. 1997. Bovine acidosis: implications on laminitis. J. Dairy. Sci. 80: 1005-1028.

SEARS, A.; WALSH, G. 1998. Industrial enzyme applications. Using these concepts to match animal enzyme and substrate in fed industry applications. In: Lyons, T.P.; Jacques, K. A. eds. Biotechnology in the Feed Industry. Proceedings of the $14^{\text {th }}$ Annual
Symposium Nottingham University Press, Loughborough, Leics, UK. p. 373-394.

SNIFFEN, C. J.; ROBINSON, P. H. 1987. Symposium: Protein and fiber digestion, passage and utilization in lactating cows. J. Dairy Sci. 70: 425-433.

STAPLES, C. R.; D. S. LOUGH. 1989. Efficacy of supplemental dietary neutralizing agents for lactating dairy cows: A review. Anim. Feed. Sci. Technol. 23: 277-303.

OETZEL, G. R. 2001. Nutritional management and subacute ruminal acidosis in dairy cattle. In: Preconvention Seminar 8: Dairy Herd Problem Investigations. American Association of Bovine Practitioners. 34th Annual Convention. September 11-12, Vancouver, B. C. 15 p.

OWENS, F. N.; SECRIST, D. S.; HILL, W. J.; GILL, D. R. 1998. Acidosis in cattle: A review. J. Anim. Sci. 76: 275-286

PEÑA, V. R. 2001. Efecto de la capacidad tampón natural de la dieta en las variables de fermentación ruminal y concentración de protozoarios en ovinos. Tesis de Maestro en Ciencias. Programa en Ganadería. Colegio de Postgraduados. Montecillo, México. 65 p.

VAN SOEST, P. J.; JONES, L. H. P. 1980. The cation exchange and buffering capacities of plant cell walls. J. Dairy Sci. 51(Supp. 1): 404 (Abstr.).

VAN SOEST, P. J.; MC BURNEY, M. I.; RUSSELL, J. 1984 Capacity exchange cation of feed for dairy rations. California Animal Nutrition Conference, Fresno. USA. 53 p.

WOHLT, J. E.; JASAITIS, K. D.; EVANS, J. L. 1987. Use of acid and base titrations to evaluate the buffering capacity of ruminant feedstuffs in vitro. J. Dairy Sci. 70: 1465-1470. 